\title{
Ultrafast X-ray study of dense-liquid-jet flow dynamics using structure-tracking velocimetry
}

\author{
YUJIE WANG ${ }^{1}$, XIN LIU ${ }^{2}$, KYOUNG-SU IM ${ }^{1}$, WAH-KEAT LEE ${ }^{1}$, JIN WANG ${ }^{1}$, KAMEL FEZZAA $^{1 *}$, \\ DAVID L. S. HUNG ${ }^{3}$ AND JAMES R. WINKELMAN ${ }^{3}$
}

\author{
${ }^{1} X-$ Ray Science Division, Argonne National Laboratory, Argonne, Illinois 60439, USA \\ ${ }^{2}$ Mayo Clinic, Rochester, Minnesota 55905, USA \\ ${ }^{3}$ Visteon Corporation, Van Buren Township, Michigan 48111, USA \\ *e-mail: fezzaa@aps.anl.gov
}

High-speed liquid jets and sprays are complex multiphase flow phenomena with many important industrial applications ${ }^{1,2}$. Great efforts have been devoted to understand their dynamics since the pioneering work of Rayleigh on low-speed jets ${ }^{3,4}$. Attempts to use conventional laser optical techniques to provide information about the internal structure of high-speed jets have been unsuccessful owing to the multiple scattering by droplets and interfaces, and the high density of the jet near the nozzle exit ${ }^{5}$. Focused-X-ray-beam absorption measurements could provide only average quantitative density distributions using repeated imaging ${ }^{6}$. Here, we report a novel approach on the basis of ultrafast synchrotron-X-ray full-field phase-contrast imaging $^{7}$. As illustrated in our case study, this technique reveals, for the first time, instantaneous velocity and internal structure of optically dense sprays with a combined unprecedented spatial and time resolution. This technique has tremendous potential for the study of transient phenomenon dynamics.

Multiphase flow is a very common phenomenon. Daily-life examples include rain, volcanoes and aerosols. The wide scientific and industrial interest in the study of multiphase flow ranges from blood flow in the human body ${ }^{8}$ to industrial liquid sprays ${ }^{9}$, and to the control of air pollution by dust particles ${ }^{10}$. Despite its mundane existence, multiphase flow can have many non-intuitive behaviours owing to the complex interactions of its different phases, such as the appearance of a sand jet when a heavy sphere is dropped on a bed of sand ${ }^{11}$. Sometimes, the importance of a phase in a multiphase phenomenon is recognized only when it is removed, as in the disappearance of splashing when a liquid drop impacts a solid surface in a vacuum environment ${ }^{12}$. Also, in the past several years, the emerging fields of microfluidics and nanofluidics have stimulated great interest in understanding complex multiphase flows in small spatial dimensions ${ }^{13,14}$.

One of the most extensively studied multiphase flows is high-speed fuel injection into a combustion chamber. A key to successfully make the combustion cleaner and more efficient is a full understanding of the breakup and atomization mechanism of the fuel jet. It has long been known that the breakup of lowspeed jets results from the unstable capillary-wave growth on the jet surface $^{15}$, but high-speed liquid-jet breakup seems to start earlier,

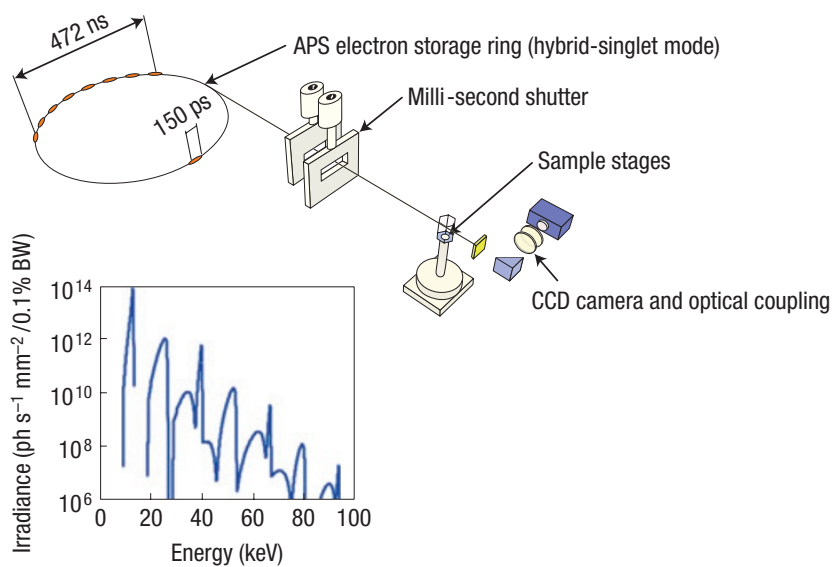

Figure 1 Schematic diagram of the experimental set-up. The X-ray beam is generated from the electron storage ring. The fill pattern shown is the hybrid-singlet mode: a single electron bunch (150 ps long and carrying $15 \mathrm{~mA}$ of current) is separated from a longer train of electrons ( $472 \mathrm{~ns}$ long, $94 \mathrm{~mA}$ ) by a $1.59 \mu \mathrm{s}$ gap on both sides. The fast shutter absorbs more than $99 \%$ of the beam power, and lets the beam through for a few milliseconds at $1 \mathrm{~Hz}$. The sample image is formed on a fast scintillator crystal (LYSO:Ce) and read on a CCD (charge-coupled device) camera via a microscope objective and a mirror at $45^{\circ}$ angle. The inset shows the APS undulator-A energy spectrum at $31 \mathrm{~mm}$ gap on a logarithmic scale. The fundamental sharp peak at $13.3 \mathrm{keV}$ is 100 times brighter than the harmonics.

at the nozzle exit ${ }^{2}$, where the distance is too short for the unstable surface wave to develop, and is strongly dependent on the nozzle internal design, initial flow conditions and jet Weber number. Several additional breakup mechanisms have been proposed, but not clearly observed ${ }^{16-18}$. Existing measurement techniques are mainly based on light scattering and suffer from absorption, reflection and multiple scattering owing to the existence of a huge number of droplets and a complex air-liquid-interface morphology when the jet is breaking up p $^{1,2,19,20}$. As a result, the dense sprays 

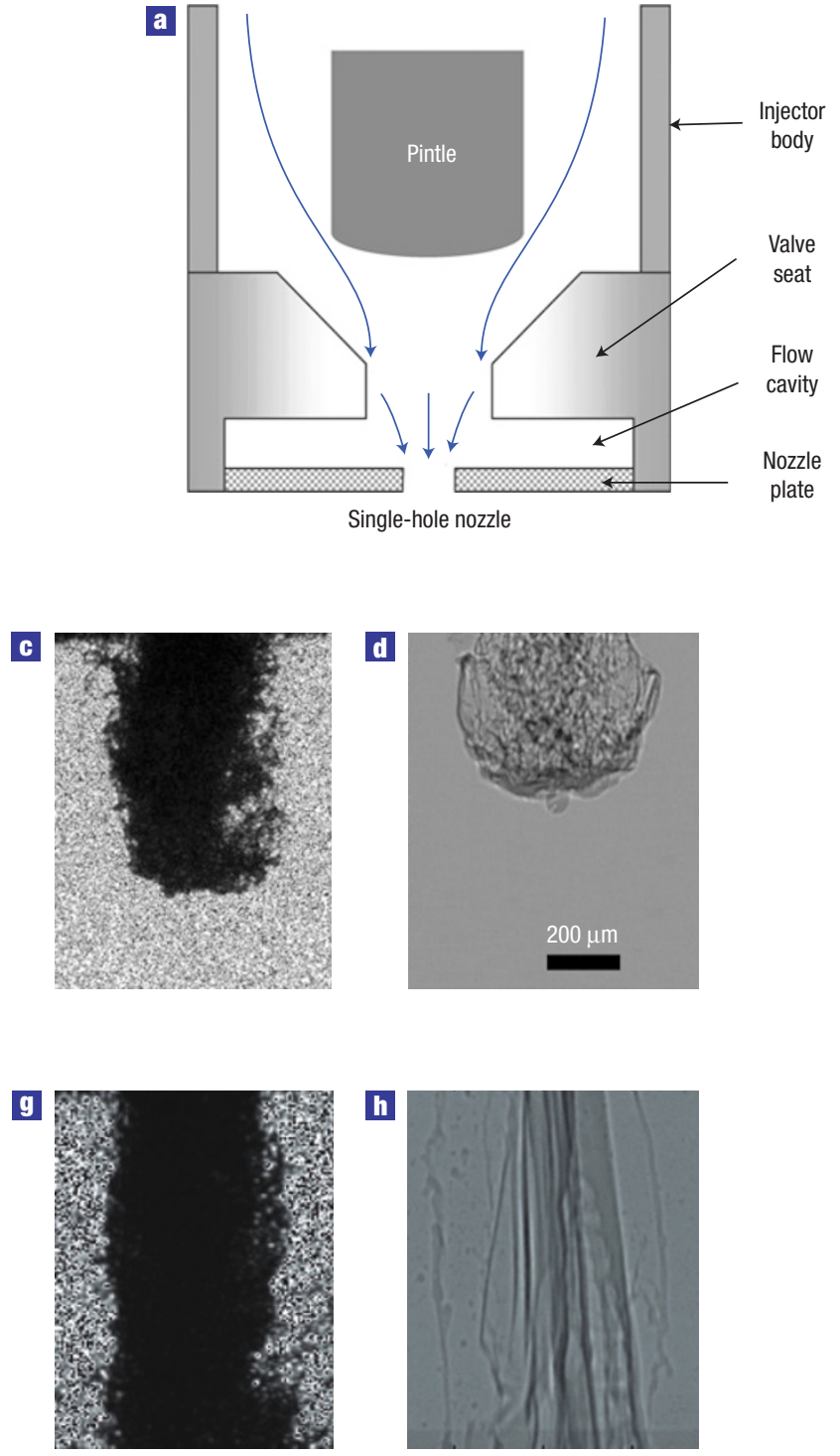

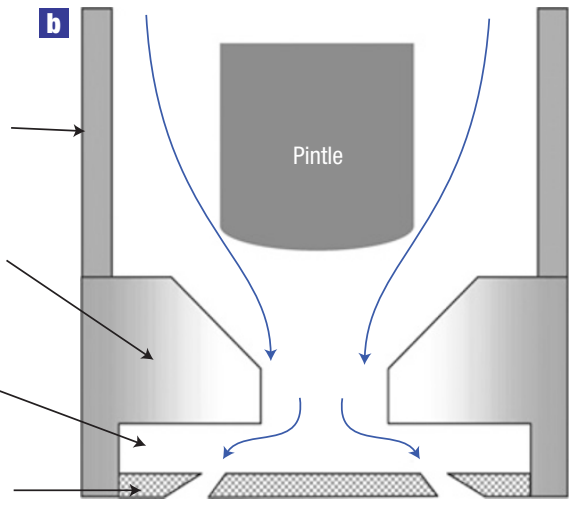

Dual-hole nozzle

e
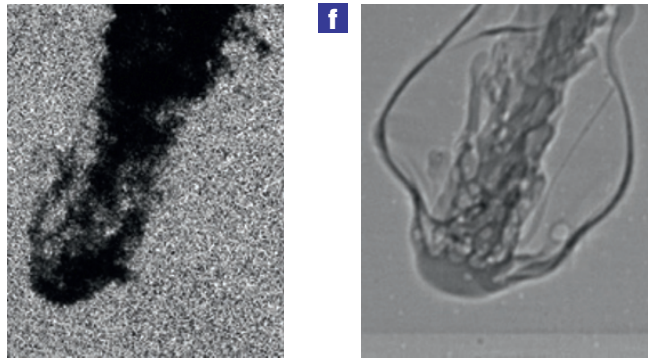

i

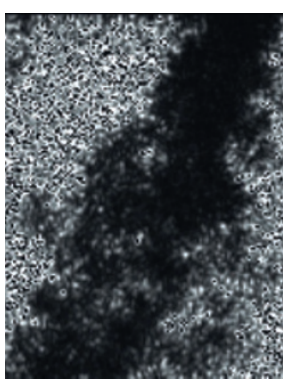

j

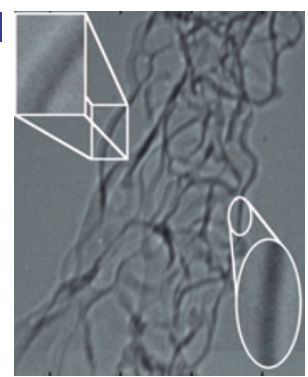

Figure 2 X-ray versus visible-light snapshots of two different types of spray. a,b, Schematic diagrams of a single-hole ( $340 \mu \mathrm{m}$ in diameter) and a dual-hole nozzle with corresponding internal flow structure respectively. The conical orifices of the dual-hole nozzle have a 340 (250) $\mu \mathrm{m}$ outer (inner) diameter respectively. The spray duration was set to $4 \mathrm{~ms}$. c,e, Shadowgraph images taken $20 \mu$ s after SOl with a 10-ns-pulsed Nd:YAG laser, near the exit of the single- and dual-hole nozzles respectively. $\mathbf{g , i}$, Shadowgraphs at the same respective locations, $1.5 \mathrm{~ms}$ after $\mathrm{SOl}$ (steady state). In all these images, only the shadows of the sprays are visible, owing to strong absorption and scattering. d,f,h,j, Normalized single-shot (472 ns) X-ray phase-contrast images of the spray in the same conditions as in c,e,g,i respectively. The X-ray images clearly show the difference between the sprays by revealing their internal structures. The insets in $\mathbf{j}$ highlight some edge enhancement effects as discussed in the text.

appear optically opaque and quantitative analysis of the images is difficult, if not impossible $\mathrm{e}^{1,2,15,21}$. We used X-rays in our previous work on coaxial water jets to circumvent some of the lightscattering difficulties, owing to the weak interaction of X-rays with matter, but no dynamical study was then feasible and our results were limited to an averaged three-dimensional watervolume-fraction reconstruction ${ }^{9}$. We have also shown that X-ray imaging can be advantageous in tracking polydispersed particles in a creeping pipe flow ${ }^{22}$. However, the use of tracer particles to map fluid velocities is an invasive approach because particles modify flow properties (especially in confined spaces), and their speeds often do not match the actual flow velocity owing to parameters such as buoyancy, particle interactions and shapes. As far as we know, there has not been any experimental measurement on the time-dependent internal morphology or velocity fields in highly turbulent and optically dense jets. Computational fluid dynamics simulation is widely adopted in fluid-mechanics research. However, owing to a dearth of experimental data to validate the initial and boundary conditions, and the complexity of these systems, current models are empirical, often oversimplified and mostly not validated by experiments ${ }^{23}$. Without information on the time-dependent morphology of the jet, it is not possible to either probe its speed or understand its dynamics and its subsequent breakup. Research in this area has been in a predicament for a long time, and there is a great need for accurate experimental measurement to serve as the input for further theoretical study.

In this paper, we introduce ultrafast X-ray phase-contrast imaging to study multiphase flow dynamics without the use 
of tracer particles, and present the first measurements on the time-dependent morphology and velocity fields of high-speed and highly turbulent jets generated by a gasoline direct injection (GDI) system. In this technique, the image contrast comes from boundaries and interfaces between materials with different refraction index or abrupt thickness variations ${ }^{7}$. The very features that hinder light-scattering experiments are at the basis of our technique.

Our experimental set-up is shown in Fig. 1, and details are given in the Methods section. GDI nozzles were developed to generate a well-atomized spray with smaller penetration using a high-turbulence nozzle concept ${ }^{24}$. The reduced penetration and small droplet sizes are particularly important in GDI systems, where the space and time for evaporation and mixing of the fuel is less than that of the ordinary port fuel injection systems. The GDI nozzle used in our study (Fig. 2) has an interchangeable nozzle plate that allows for different geometries of exit orifices. For our single-hole nozzle plate, the fuel exits the nozzle through the orifice in a straight downward direction. In contrast, the orifice locations of the dual-hole nozzle plate are off-centred and are intended to induce 'eddies' by forcing the liquid to flow around the sharp corners of the valve seat before reaching the orifices. It has been speculated, and verified experimentally, that the expected introduction of eddies or turbulence, once trapped in the main jet, can cause an early breakup or soft spray in the downstream region $^{24-26}$. However, the differences of the two spray structures in the near-nozzle region, where the nozzle design effect is more predominant, could not be determined by visible-light imaging measurements, owing to their optical opacity (Fig. 2c,e,g,i). This is a major problem in these systems: even though there has been ample evidence downstream (where breakup can be measured using visible-light techniques) to infer the nozzle effect indirectly, how exactly the internal turbulence, cavitation or simple velocity rearrangement influence the breakup remains unknown. In sharp contrast, the high-speed X-ray images in Fig. 2d,f and h,j show clear differences between the internal structures of the two sprays. The characteristic 'sac', leading the jet, is clearly visible in the $\mathrm{X}$-ray images. It corresponds to the static liquid trapped in the flow cavity. It is simply pushed out after the start of injection (SOI), and undergoes very little turbulence inside the nozzle. It also moves more slowly than the subsequent spray (see later). Images in Fig. $2 \mathrm{~g}-\mathrm{j}$ represent the steady flow at $1.5 \mathrm{~ms}$ after SOI. The jet from the straight-hole nozzle has a smooth and fairly unperturbed morphology, whereas the jet from the doublehole nozzle possesses a distinctive complex morphology. A close examination of the X-ray image in Fig. $2 \mathrm{j}$ shows that the spray is dominated by a membrane-type network at the exit of the nozzle. The bright-dark pair of edges (X-ray refraction effect) in the magnified rectangular inset indicates a thick convex membrane, and the dark stripe with bright edges (elliptical inset) corresponds most likely to the rim of a thinned membrane. A detailed study is beyond the scope of this letter, but this membrane-mediated breakup has been observed in other systems ${ }^{27}$. The existence of this complex morphology at the exit of the nozzle provides the first direct confirmation that the earlier jet breakup in the dualhole GDI nozzle is indeed due to the instabilities created within the nozzle itself. It also explains the soft-spray smaller volume fraction recently measured using X-ray absorption ${ }^{26}$. Because the complex membrane structures extend spatially into the gas environment, gas is entrained inside the jet, which leads naturally to a smaller volume fraction. We emphasize that this complex morphology in the near nozzle region cannot be directly seen with any other technique. These dynamic effects and texture differences are even more striking in the recorded movies of the sprays (see the Supplementary Information).
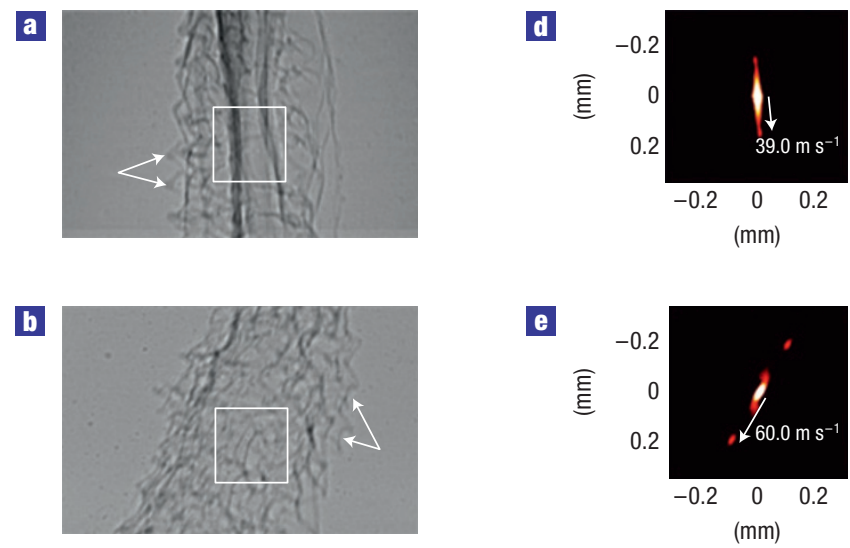

G

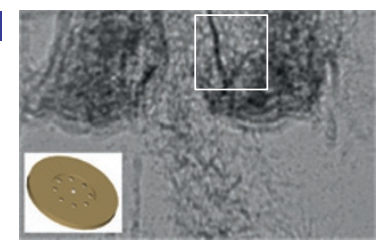

f

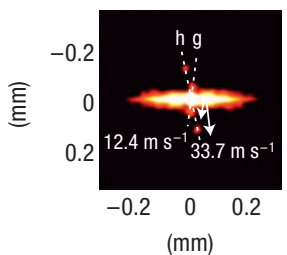

g

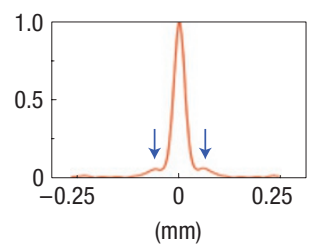

h

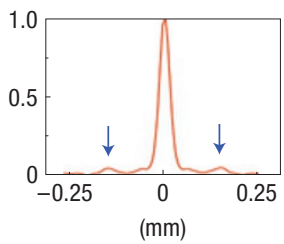

Figure 3 Velocity measurements by autocorrelation. a-c, X-ray double-exposure images (472 ns exposure time, $3.68 \mu$ s shot-to-shot delay) of three different types of spray structure, from a single-hole, dual-hole and nine-hole nozzle respectively. The inset in $\mathbf{c}$ shows the interchangeable nine-hole nozzle plate. $\mathbf{d}-\mathbf{f}$, Autocorrelation functions of the corresponding interrogation windows $\left(0.34 \times 0.34 \mathrm{~mm}^{2}\right)$ marked as boxes in a-c respectively. The velocities are calculated on the basis of the peak positions in the autocorrelation functions. Panel $\mathbf{c}$ was chosen in a complex (two-) overlapping-spray structure. The autocorrelation $\mathbf{f}$ shows two pairs of peaks corresponding to two overlapping sprays with different vector velocities. This demonstrates that $\mathrm{X}$-rays can pick up both velocity components even though one spray is spatially totally blocked. $\mathbf{g}, \mathbf{h}$, Normalized line-cuts across the autocorrelation in $\mathbf{f}$ showing the two peaks.

By taking advantage of the X-ray pulse time structure and the clarity of the spray texture in the X-ray images, we have been able to extract velocity fields without the need for particle seeding. The velocity fields of the liquid spray in the breakup phase were measured by tracking the movements of its phaseenhanced texture related to the liquid-gas boundaries, using local autocorrelation analysis of the double-exposed images. In addition to the single- and dual-hole nozzles, we also investigated a ninehole nozzle. Because we are mostly interested in the centre of the spray, where visible-light techniques are not applicable, the autocorrelation interrogation windows were chosen accordingly, as marked out by boxes in Fig. 3a-c. The corresponding intensity autocorrelations of the interrogation windows are plotted in Fig. 3d-f. For all three sprays, there exist a bright central peak that corresponds to the self-correlation of the image and pairs of symmetric peaks that correspond to the correlation of the original image with the displaced image after the interval of the double exposure. The instantaneous velocity of the corresponding sprays, 

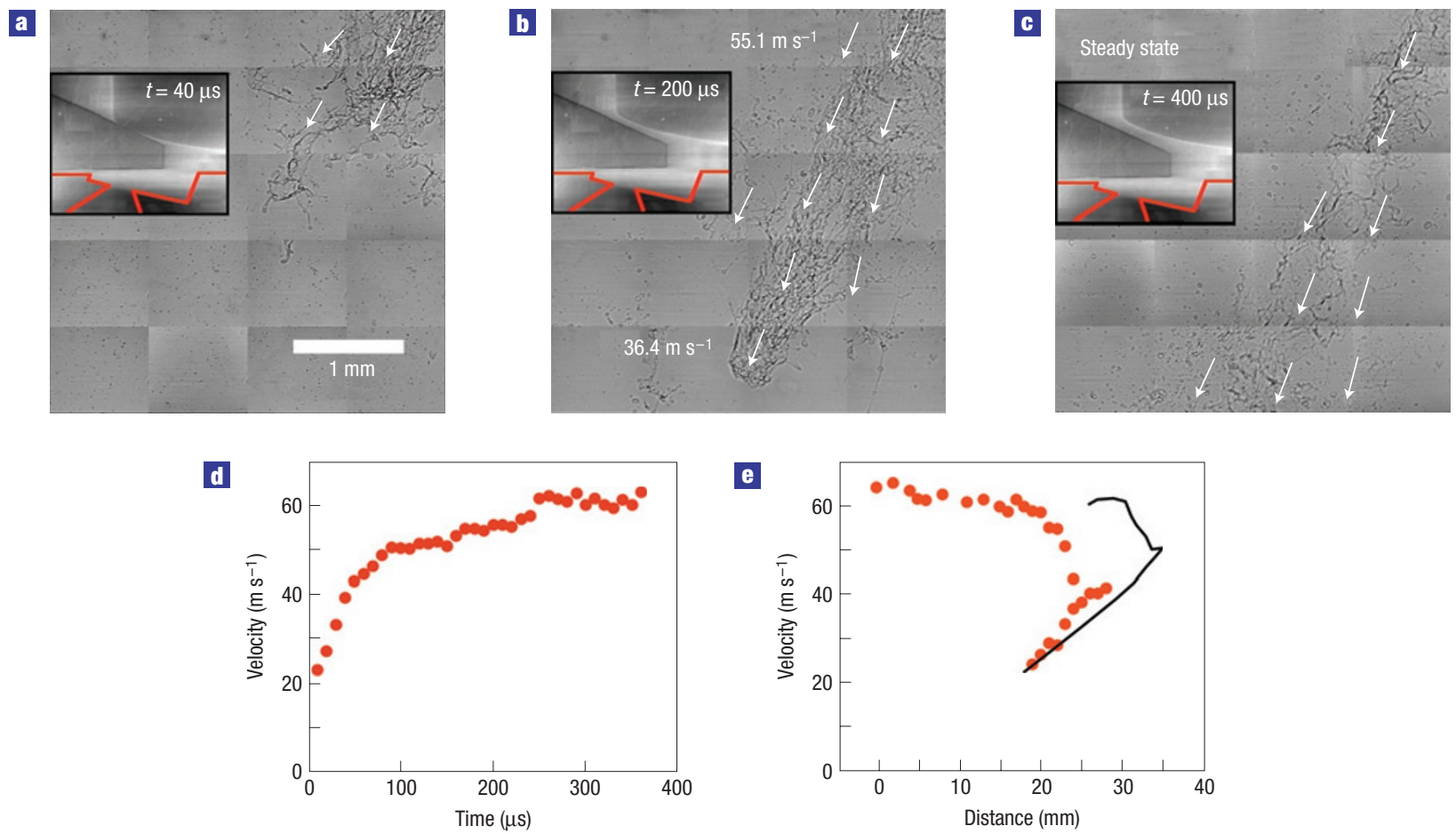

\begin{abstract}
Figure 4 Dynamics of the velocity field. a-c, Velocity maps of the jet at $t=40,200$ and $400 \mu \mathrm{s}$ after SOl. Insets, High-energy phase-contrast images (20 $\mu$ s exposure time) of the pintle moving inside the dual-hole nozzle. $\mathbf{d}$, Spray velocity near the nozzle exit as a function of time since the emergence of the jet. The time for the jet to reach steady-state velocity is equal to the pintle opening time (about $300 \mu \mathrm{s}$ ). e, Spray velocity (red circles) as a function of spatial distance from the nozzle exit up to $30 \mathrm{~mm}$, at $780 \mu \mathrm{s}$ after SOI. From 19 to $24 \mathrm{~mm}$, velocities of two spatially overlapping components are measured. The solid line indicates the calculated velocity and position of the jet, on the basis of data from $\mathbf{d}$ (see text).
\end{abstract}

averaged over the interrogation box, can readily be deduced. The narrow width of the correlation peaks in Fig. 3e indicates that the velocity distribution is small $\left(60 \pm 5 \mathrm{~m} \mathrm{~s}^{-1}\right.$ at FWHM), and the contribution (to the measured mean velocity) of the dispersively moving features such as the outer droplets is negligible compared to the spray core. Furthermore, Fig. 3f clearly shows that the velocity of different overlapping parts of the spray can be differentiated. The angle between the two velocity vectors is consistent with the nozzle design specifications. Line scans across the autocorrelation peaks (Fig. $3 \mathrm{~g}, \mathrm{~h}$ ) demonstrate the good statistics in finding the autocorrelation peaks, even though the interrogation window was chosen inside a very dense area of the spray. The spatial resolution in extracting the velocity field, defined as the smallest interrogation window leading to autocorrelation peaks with a sufficient signal-tonoise ratio, was empirically estimated to be $300 \mu \mathrm{m}$ in this study.

To gain a better understanding of how this initially perturbed jet will eventually disintegrate into droplets, we tracked the temporal and spatial evolution of the jet and its velocity from a dual-hole nozzle. The snapshots were taken at different times $t$ after SOI. Figure $4 \mathrm{a}, \mathrm{b}$ corresponds to the acceleration phase $(t=40 \mu \mathrm{s}$ and $200 \mu$ s respectively), and Fig. $4 \mathrm{c}$ to a steady state $(t=400 \mu \mathrm{s})$. The on-axis near-nozzle jet velocity rapidly reaches $75 \%$ of its maximum velocity within $40 \mu \mathrm{s}$ after SOI, and reaches its maximum steady-state velocity $\left(60 \mathrm{~m} \mathrm{~s}^{-1}\right)$ at about $250 \mu$ s after SOI (Fig. $4 \mathrm{~d}$ ). To correlate the spray dynamics with the injection cycle, we imaged the pintle motion (Fig. $4 \mathrm{a}-\mathrm{c}$ insets) using the same imaging technique, but with high-energy $\mathrm{X}$-rays (see the Methods section). The time it takes to achieve steady-state velocity is consistent with the opening time of the pintle. The spray velocity as a function of distance from the nozzle, at $780 \mu$ s after SOI, is shown in Fig. 4e. Between 19 and $24 \mathrm{~mm}$, the autocorrelation shows two distinct peaks. In this region, the fast portion of the jet, which exits the nozzle at $\sim 200-300 \mu$ s after SOI, has caught up with the slower portion of the jet, which exits the nozzle immediately after SOI. This interpretation is qualitatively supported by simple free-flow (ignoring drag, coalescence, breakup and collision) calculation (Fig. 4e, line) on the basis of the near-nozzle measurements in Fig. 4 d. These measurements clearly point to the inadequacy of conventional techniques that only yield the leading-edge velocity ${ }^{28}$.

\section{METHODS}

The experiment was carried out at XOR 32-ID beamline of the Advanced Photon Source (APS) at Argonne National Laboratory, Illinois. The undulator source provided the high X-ray brilliance necessary for this white-beam ultrafast imaging technique. With the undulator gap set to $31 \mathrm{~mm}$, most of the intensity was located within the first harmonic at $13.3 \mathrm{keV}$, with a peak irradiance of $10^{14} \mathrm{ph} \mathrm{s}^{-1} \mathrm{~mm}^{-2} / 0.1 \% \mathrm{bw}$, and a natural bandwidth of $0.3 \mathrm{keV}$ full-width at half-maximum (FWHM). For imaging of the pintle motion (Fig. 4a-c insets), the higher-order harmonic was used (at $66 \mathrm{keV}$ ), and the lower energy was filtered with a $10-\mathrm{mm}$-thick silicon slab. The sample stage was located $40 \mathrm{~m}$ downstream from the source. A fast-scintillator crystal (LYSO:Ce, with a 40 ns decay time) converted the transmitted X-rays into visible light $(434 \mathrm{~nm})$. The sample-scintillator distance, set to $700 \mathrm{~mm}$, corresponded to an optimized defocus value for Fresnel propagation contrast and spatial resolution. The images were captured with a fast CCD camera (Sensicam HS-SVGA, $1,024 \times 1,280$ pixels, from Cooke) coupled to the scintillator via a microscope objective $(\times 5, \mathrm{NA}=0.14)$ and a $45^{\circ}$ mirror. The field of view of the imaging system $\left(1.36 \times 1.71 \mathrm{~mm}^{2}\right)$ matched the full usable X-ray-beam size. The spatial resolution was determined by both the intrinsic imaging resolution (point spread function $\sim 2 \mu \mathrm{m}$ FWHM) and the motion smearing of the moving subject, which yielded a combined resolution of several micrometres in current experiments $(5-30 \mu \mathrm{m})$. The shutter, CCD camera and injection system were synchronized to the X-ray pulses using Stanford DG535 delay generators fed 
with the radio-frequency signal from the storage ring. Visible-light images (Fig. 2c,e,g,i) were obtained by backlighting the sprays using a Nd:YAG laser with $10 \mathrm{~ns}$ pulse duration (SprayMaster System, LaVision). We used a simulated fuel (Viscor 16-A) that has physical properties formulated to be similar to gasoline fuel but less flammable. The fuel was injected into a quiescent nitrogen environment at $0.1 \mathrm{MPa}$. The injection pressure was set to $2 \mathrm{MPa}$. The nozzles were custom made for Visteon.

A critical aspect of this technique is the X-ray time structure. The APS operates with an electron storage ring period of $3.68 \mu$ s and a harmonic number of 1,296 (ref. 29). A variety of different ring fill patterns are available, of which a 'hybrid-singlet' mode is most relevant to our ultrafast imaging (Fig. 1). Although our fast electronics and shutters are capable of isolating and synchronizing to either bunch to capture images, all images in this paper were obtained from the $472 \mathrm{~ns}$ 'superbunch'.

Received 4 September 2007; accepted 21 December 2007; published 27 January 2008.

\section{References}

1. Reitz, R. D. \& Bracco, F. V. The Encyclopedia of Fluid Mechanics Vol. 3, 233-249 (Gulf Publishing, New Jersey, 1986).

2. Reitz, R. D. \& Bracco, F. V. Mechanism of atomization of a liquid jet. Phys. Fluids 25, $1730-1742$ (1982)

3. Lin, S. P. \& Reitz, R. D. Drop and spray formation from a liquid jet. Annu. Rev. Fluid Mech. 30, 85-105 (1998).

4. Rayleigh, W. S. On the stability of jets. Proc. Lond. Math. Soc. 4, 10-13 (1878).

5. Chigier, N. \& Reitz, R. D. in Recent Advances in Spray Combustion: Spray Atomization and Drop Burning Phenomena Vol. 1 (ed. Kuo, K. K.) 109-135 (AIAA, Reston, 1996).

6. MacPhee, A. G. et al. X-ray imaging of shock waves generated by high-pressure fuel sprays. Science 295, 1261-1263 (2002)

7. Wilkins, S. W., Gureyev, T. E., Gao, D., Pogany, A. \& Stevenson, A. W. Phase-contrast imaging using polychromatic hard X-rays. Nature 384, 335-338 (1996).

8. Milnor, W. R. Hemodynamics 2nd edn, 5 (Williams \& Wilkins, Baltimore, 1989).

9. Wang, Y. J. et al. Quantitative $\mathrm{x}$-ray phase-contrast imaging of air-assisted water sprays with high Weber numbers. Appl. Phys. Lett. 89, 151913 (2006).

10. Brennen, C. E. Fundamentals of Multiphase Flow 1-2 (Cambridge Univ. Press, New York, 2005).

11. Royer, J. R. et al. Formation of granular jets observed by high-speed X-ray radiography. Nature Phys. 1, 164-167 (2005)
12. Xu, L., Zhang, W. W. \& Nagel, S. R. Drop splashing on a dry smooth surface. Phys. Rev. Lett. 94, 184505 (2005)

13. Whitesides, G. W. The origins and the future of microfluidics. Nature 442, 368-373 (2006).

14. Squires, T. M. \& Quake, S. R. Microfluidics: Fluid physics at the nanoliter scale. Rev. Mod. Phys. 77, 977-1026 (2005).

15. Donnely, R. J. \& Glaberson, W. Experiment on the capillary instability of a liquid jet. Proc. R. Soc. Lond. A 290, 547-556 (1965).

16. Schweitzer, P. H. Mechanism of disintegration of liquid jets. J. Appl. Phys. 8, 513-521 (1937)

17. Bergwerk, W. Flow pattern in diesel nozzle spray holes. Proc. Inst. Mech. Eng. 173, 655-660 (1959).

18. Rupe, J. H. On the dynamic characteristics of free liquid jets and a partial correlation with orifice geometry. NASA JPL Technical Report No. 32, 207-240 (1962).

19. Voges, H. et al. Proceedings of the 5th Conference of ILASS-Asia, Annual Conference on Liquid Atomization and Spray Systems 35-42 (2000).

20. Friedlander, S. K. Smoke, Dust, and Haze: Fundamentals of Aerosol Dynamics 2nd edn, 136 (Oxford Univ. Press, New York, 2000).

21. Meyers, J. F. \& Komine, H. in Laser Anemometry-Advances and Applications Vol. 1 (eds Dybbs, A. \& Ghorashi, B.) 289-296 (ASME Publications, Cleveland, 1991).

22. Im, K.-S. et al. Particle tracking velocimetry using fast $\mathrm{x}$-ray phase-contrast imaging. Appl. Phys. Lett. 90, 091919 (2007).

23. Reitz, R. D. Modeling atomization process in high-pressure vaporizing sprays. Atomization Spray Technol. 3, 309-337 (1987).

24. Xu, M. et al. Soft spray formation of a low-pressure, high-turbulence fuel injector for direct-injection gasoline engines. SAE Trans. J. Fuels Lubricants 111, 1452-1466 (2002).

25. Zhao, F. Q., Harrington, D. L. \& Lai, M. C. Automotive Gasoline Direct-Injection Engines 65 (Society of Automotive Engineers, Warrendale, April 2002).

26. Liu, X. et al. Quantitative characterization of near-field fuel sprays by multi-orifice direct injection using ultrafast $\mathrm{x}$-tomography technique. SAE Trans. J. Eng. 115, 576-583 (2006).

27. Lasheras, J. C. \& Hopfinger, E. J. Liquid jet instability and atomization in a coaxial gas stream. Annu. Rev. Fluid Mech. 32, 275-308 (2000).

28. Sedarsky, D. L., Paciaroni, M. E., Linne, M. A., Gord, J. R. \& Meyer, T. R. Velocity imaging for the liquid-gas interface in the near field of an atomizing spray: Proof of concept. Opt. Lett. 31, 906-908 (2006).

29. Borland, M. et al. APS storage ring parameters. http://aps.anl.gov/Facility/Storage_Ring_Parameters (2007)

\section{Acknowledgements}

The use of the APS was supported by the US Department of Energy, Office of Science, Office of Basic Energy Sciences, under contract DE-AC02-06CH11357, and Argonne National Laboratory Director's Competitive Grant (LDRD) 2006-023-N0.

Correspondence and requests for materials should be addressed to K.F.

Supplementary Information accompanies this paper on www.nature.com/naturephysics.

Reprints and permission information is available online at http://npg.nature.com/reprintsandpermissions 